\title{
Oxycodone vs Sufentanil in Patient-Controlled Intravenous Analgesia After Gynecological Tumor Operation: A Randomized Double-Blind Clinical Trial
}

This article was published in the following Dove Press journal: Journal of Pain Research

\author{
Sha-Jie Dang ${ }^{1,2, *}$ \\ Rui-Li Li ${ }^{3, *}$ \\ Jun Wang' \\ Wen-Bin Zeng ${ }^{\prime}$ \\ Yun $\mathrm{He}^{\mathrm{I}}$ \\ Hui-Yu Yuel \\ Si-Yuan $\mathrm{Li}^{4}$ \\ Li-Chun $\operatorname{Han}^{2,5}$ \\ 'Department of Anesthesia, Shaanxi \\ Provincial Cancer Hospital, Xi'an, \\ Shaanxi, People's Republic of China; ${ }^{2}$ The \\ Key Laboratory of Biomedical \\ Information Engineering of Ministry of \\ Education, School of Life Science and \\ Technology, Xi'an Jiaotong University, \\ Xi'an, Shaanxi, People's Republic of \\ China; ${ }^{3}$ Department of Pharmacy, Xijing \\ Hospital, Fourth Military Medical \\ University, Xi'an, Shaanxi, People's \\ Republic of China; ${ }^{4}$ Department of \\ Anesthesia, The Second Affiliated \\ Hospital of Xian Jiaotong University, \\ Xi'an, Shaanxi, People's Republic of \\ China; ${ }^{5}$ Department of Anesthesia, Xi'an \\ Daxing Hospital, Xi'an, Shaanxi, People's \\ Republic of China \\ *These authors contributed equally to \\ this work
}

Correspondence: Li-Chun Han Department of Anesthesia, Xi'an Daxing Hospital, Xi'an, Shaanxi 7I00I6, People's Republic of China

Email hanlichun696@163.com

Si-Yuan Li

Department of Anesthesia, The Second Affiliated Hospital of Xian Jiaotong

University, Xi'an, Shaanxi 7I0004,

People's Republic of China

Email foxlsy@।63.com
Background: This study aims to compare analgesic effect and side effects of oxycodone and sufentanil in transition analgesia and patient-controlled intravenous analgesia (PCIA) after gynecological tumor operation under general anesthesia.

Patients and Methods: A prospective, randomized, double-blind research was conducted. Patients undergoing elective gynecological tumor surgery were randomized into four groups: Group S (sufentanil transition analgesia and sufentanil PCIA), Group OS (oxycodone transition analgesia and sufentanil PCIA), Group SO (sufentanil transition analgesia and oxycodone PCIA) and Group O (oxycodone transition analgesia and oxycodone PCIA). The primary outcomes were Numerical Rating Scale (NRS) at rest and coughing, accumulated opioid consumption in PCIA and patients' satisfaction.

Results: Patients in Group OS and Group O showed shorter time of consciousness recovery and extubation after surgery. Accumulated opioid consumption in PCIA (equal to morphine) in Group SO and Group O was significantly less than that in Group S and Group OS. Patients in Group O showed lower NRS at rest and coughing, but higher patients' satisfaction 3, 24 and 48 hours after surgery. Patients in Group SO and Group O showed a shorter time of intestinal recovery, first feeding and first-time movement.

Conclusion: Both oxycodone and sufentanil provided adequate pain relief in transitional analgesia and PCIA treatment after surgery. Oxycodone without background infusion showed less analgesic drug consumption and faster recovery than sufentanil with background infusion in PCIA after gynecological tumor operation under general anesthesia.

Keywords: gynecological tumor operation, oxycodone, transition analgesia, patientcontrolled intravenous analgesia, sufentanil

\section{Introduction}

Gynecological tumor operation is often accompanied with severe postoperative pain, which can cause atelectasis, sympathetic over activity, prolonged hospital staying, and poor patient's satisfaction. Furthermore, unrelieved severe pain may lead to chronic pain, that seriously affects patients' quality of life. ${ }^{1,2}$ Patientcontrolled intravenous analgesia (PCIA) is known to provide an efficient way for postoperative pain relief. ${ }^{3}$ There are two different administration patterns in PCIA including background infusion and no background infusion. In recent published guideline of postoperative analgesia, ${ }^{4}$ no background infusion in PCIA was recommended due to the potential association between background opioid infusion and adverse complication, especially respiratory depression. ${ }^{5}$ 
Opioids are considered to be the standard analgesic to relieve moderate to severe acute postoperative pain. Morphine is widely used in postoperative analgesia management in Europe and North America, whereas sufentanil and fentanyl are more popular in China because of their quicker onset of action and less adverse effects especially respiratory depression and postoperative nausea \& vomiting (PONV). Oxycodone, a semisynthetic opioid which can bind to both $\mu$-and $\kappa$-opioid receptors, ${ }^{6}$ was widely used in intravenous injection for postoperative analgesia in China since the approval in 2013. It has been reported that intravenous injection of oxycodone can provide satisfactory postoperative analgesia by several studies. ${ }^{7-14}$ Considering the pivotal role of $\kappa$-opioid receptors in visceral pain attenuation, it is reasonable to hypothesize that oxycodone can enhance the quality of analgesia. Moreover, the half-life of oxycodone is 3.5 hours, it is more suitable to no background infusion in PCIA, which may reduce the side effects. However, there are few reports investigating oxycodone PCIA without background infusion.

In this study, a randomized, double-blind clinical trial was performed to evaluate analgesic effects and side effects of oxycodone in transition analgesia and PCIA after gynecological tumor operation under general anesthesia.

\section{Patients and Methods Study Design}

A prospective, randomized and double-blind study was conducted at the departments of anesthesia in Shaanxi Provincial Cancer Hospital between January 2017 and June 2018. Written informed consent was obtained from each of the patient, otherwise their next of kin or their legal representative. This study was approved by the clinical research ethics committee of Shaanxi Provincial Cancer Hospital, and was registered in Chinese Clinical Trial Registry (registration number ChiCTR-IOR-1800014793). This study followed the Good Clinical Practice guidelines and the guidelines of the Helsinki Declaration.

\section{Patients}

Patients (aged 24 64 years old, BMI 18.1 31.1 and ASA I II) undergoing elective gynecological tumors operation (laparotomy or endoscopy) under general anesthesia were screened in this study. The following patients were excluded: 1) Allergy, sensitivity or contraindication to study medications. 2) Inability to communicate in the preoperative period (coma, profound dementia, or language barrier). 3) Severe hypertension (systolic blood pressure $\geq 180 \mathrm{mmHg}$, diastolic blood pressure $\geq$ $110 \mathrm{mmHg}$ ). 4) Brain injury or neurosurgery. 5) History of severe hepatic, renal, pulmonary, or cardiac disease. 6) Preoperative history of schizophrenia, epilepsy, Parkinsonism or myasthenia gravis. 7) History of chronic opioid usage within eight weeks of surgery. 8) Taking monoamine oxidase inhibitors within two weeks before the surgery. 9) Pregnant and lactation women. 10) Others that is not suitable for application.

\section{Randomization and Masking}

A biostatistician, who was independent of data management and statistical analyses, generated random numbers (in a 1:1 ratio) using the SAS 9.2 software (SAS Institute, Cary, NC). The results of randomization stored online (https://pan.baidu.com) until the end of the study.

There were 124 patients undergoing elective gynecological tumors surgery who were randomized into four groups: Group $\mathrm{S}$ (sufentanil transition analgesia and sufentanil PCIA, $\mathrm{n}=32$ ), Group OS (oxycodone transition analgesia and sufentanil PCIA, $n=30$ ), Group SO (sufentanil transition analgesia and oxycodone PCIA, $n=30$ ) and Group O (oxycodone transition analgesia and oxycodone PCIA, $\mathrm{n}=32$ ). Their anesthesiologist login in online and administered the study drugs according to the randomization sequence, which was marked as red color after usage. Study personnel, health-care team members, and patients were masked to the group assignment throughout the study period. In an emergency, unmasking of the treatment allocation could be requested, and the study would be terminated. These situations were documented and analyzed to reveal its potential association with the treatment.

\section{Dose Determination}

The equivalent dose converting between parenteral morphine and oxycodone is variable, with a suggested ratio of 0.65 to 1.5. ${ }^{15}$ In this study, the ration of 1 was chosen on the assumption that 1 dose of oxycodone may be equal to morphine according to the recently published literatures. ${ }^{9,15}$ The equivalent dose converting between sufentanil and morphine was 0.001 . Therefore, the equivalent dose converting between sufentanil and oxycodone was 0.001 .

\section{Procedures}

Patients were not given any sedative, analgesic, antemetic, or anti-itching drugs 24 hours before the operation. Patients were fasting from solids and liquids 8 hours before the operation. The purpose of this study, PCIA instruction, numeric rating scale (NRS) calculation method 
and side effects were explained to the patients one day before the surgery. After arriving the operation room, routine monitoring including electrocardiogram (ECG), pulse oximetry $\left(\mathrm{SpO}_{2}\right)$, heart rate $(\mathrm{HR})$, noninvasive blood pressure (BP) and bispectral index (BIS) were established prior to the anesthesia.

General anesthesia was conducted with $0.05 \mathrm{mg} / \mathrm{kg}$ midazolam, $0.4 \mu \mathrm{g} / \mathrm{kg}$ sufentanil, $0.2 \mathrm{mg} / \mathrm{kg}$ etomidate, and 0.2 $\mathrm{mg} / \mathrm{kg}$ cisatracurium. Total intravenous general anesthesia was used in this study: cisatracurium was injected continuously $(0.1 \mathrm{mg} / \mathrm{kg} \cdot$ hour$)$ to maintain muscle relaxation; the maintenance doses of propofol and remifentanil $(0.1 \sim 0.3 \mu \mathrm{g} / \mathrm{kg} \cdot \mathrm{min})$ were adjusted to keep BIS between 40 60, and hemodynamic alteration between $20 \%$ compared to the baseline BP. The infusion of cisatracurium was terminated 30 minutes prior to the approximate completion of surgery. Moreover, $5 \mathrm{mg}$ dexamethasone and $0.3 \mathrm{mg}$ of tropisetron were administered in order to prevent PONV, the most common side effects after general anesthesia. Patients in Group $\mathrm{O}$ and Group OS received oxycodone $(0.1 \mathrm{mg} / \mathrm{kg}$ for endoscopy procedures or $0.15 \mathrm{mg} / \mathrm{kg}$ for laparotomy procedures), whereas patients in Group S and Group SO received sufentanil $(0.1 \mu \mathrm{g} / \mathrm{kg}$ for endoscopy procedures or $0.15 \mu \mathrm{g} / \mathrm{kg}$ for laparotomy procedures) before the end of anesthesia as transition analgesia.

After the end of anesthesia, PCIA device (Apon, ZZBI, Medical technology Corporation, Jiangsu, China) was connected to patients, and continued 48 hours for postoperative pain relief. In Group S and Group OS, a bolus dose was $2 \mu \mathrm{g}$ and background infusion was $2 \mathrm{~mL} / \mathrm{h}$, in Group SO and Group O, a bolus dose was $2 \mathrm{mg}$ and there was no background infusion. The lockout time was 5 mins for all patients. Patients were transferred into post-anesthesia care unit (PACU) after surgery. Extubation was performed after confirming the recovery of spontaneous breathing. Time of consciousness recovery and extubation after surgery were documented. Postoperative pain was evaluated with Numerical Rating Scale (NRS) 5 mins after extubation. 0 indicating no pain and 10 unbearable pain. Bonus dose $(2 \mu \mathrm{g}$ sufentanil or $2 \mathrm{mg}$ oxycodone injection) was given to the patients immediately if the NRS was more than 3. The procedures were repeated when NRS was evaluated 5 mins after bonus until the NRS was less than 4. Sedation levels, rescue analgesia and side effects in PACU were documented.

Length of first demand bolus in ward after surgery, accumulated opioid consumption of PCIA, NRS at rest and coughing, sedation level, FAS, side effects, patients' satisfaction and time of intestinal recovery, first feeding and first-time movement were evaluated and recorded 3 hours, 24 hours and 48 hours after surgery. The upper limitation was $10 \mathrm{mg}$ oxycodone or $10 \mu \mathrm{g}$ sufentanil within one hour by limiting its number of bolus times. If the consumptive analgesia drugs reached the limitation and still felt painful, the responsible anesthesiologist would be called and alternative rescues analgesia drug should be administrated and recorded.

The Ramsay sedation scale was applied to assess the sedation level ( 1 = anxious or restless or both, $2=$ cooperative, oriented, and tranquil, 3 = responds to command, $4=$ brisk response to a light glabellar tap or loud auditory stimulus, $5=$ sluggish response to a light glabellar tap or loud auditory stimulus, and $6=$ no response to the stimuli). Functional activity score (FAS), 1 indicating unrestricted activity, 2 indicating partially restricted activity and 3 indicating severely restricted activity. Patients' satisfaction was measured on a 5 point scale ( 1 = very dissatisfied, $2=$ dissatisfied, $3=$ neutral, $4=$ satisfied, and 5 = very satisfied). The side effects observed were PONV, respiratory depression, pruritus and dizziness. When $\mathrm{SpO}_{2}$ was $<90 \%$ and respiration rate was $<10$ breath/ min, it was documented as respiratory depression.

\section{Outcomes}

Outcome assessment was completed by research members who were trained before the study and not involved in the clinical care of the patients. The primary outcomes were rescued analgesia rate in PACU, NRS at rest and coughing, accumulated opioid consumption in PCIA and patients' satisfaction. The secondary outcomes included time of consciousness recovery and extubation, length of first demand bolus in ward after surgery, sedation level, FAS, side effects and time of intestinal recovery, first feeding and first-time movement.

\section{Statistical Analysis}

The primary endpoint was the NRS at rest over time. There was 20\% 33\% decrease in VAS with respect to previous studies, ${ }^{16}$ in the preliminary study, 20 patients were assigned to Group S, Group OS, Group SO and Group $\mathrm{O}(\mathrm{n}=5)$, and the NRS at rest 24 hours after surgery were $4.0 \pm 2.2,3.5 \pm 1.9,2.0 \pm 1.5$ and $2.5 \pm 1.8$, respectively. A sample size of 29 per group was obtained by PASS 11.0 (NCSS, LLC, Kaysville, Utah, USA) with two-tailed $\alpha=$ 0.05 and $\beta=0.90$. Take into account a dropout rate of approximately $20 \%$, we planned to enroll 35 patients for each group.

The statistical analysis was performed with SPSS 24.0 for Windows (SPSS, Inc., IBM) and Graphpad Prism 7 


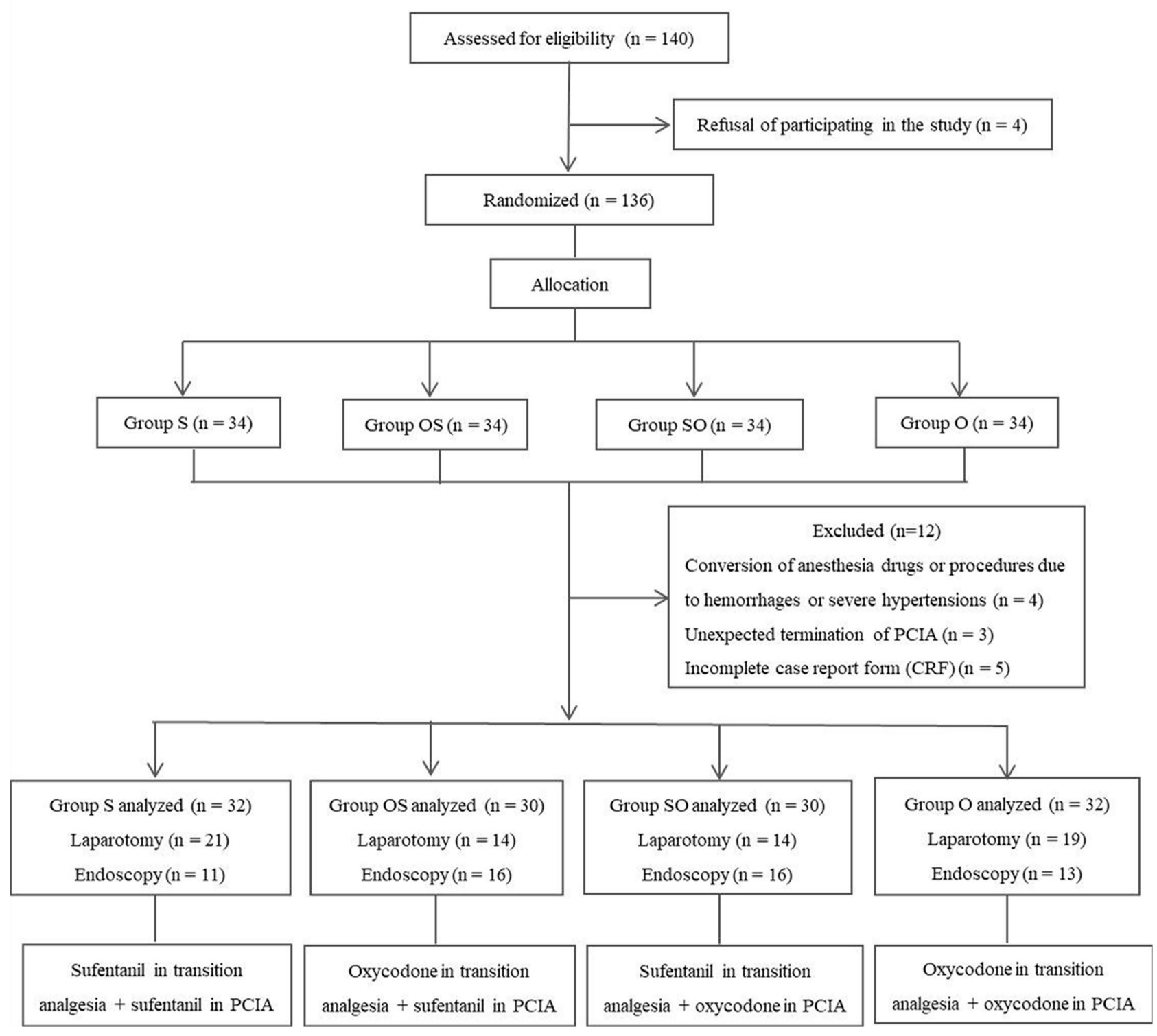

Figure I Consolidated standards of reporting trials (CONSORT) flow diagram.

Note: Data analysis included all patients in the groups to which they were randomly assigned.

Abbreviation: PCIA, patient-controlled intravenous analgesia.

(GraphPad Software, La Jolla, CA, USA). Measured data with normal distributions are expressed as Mean $\pm \mathrm{SD}$, nonnormally distributed data are expressed as Median (P25, P75). ANOVA was used if data were normal distributions and the variations were homogenous, otherwise Wilcoxon rank-sum test was used. A repeated measure analysis of variance (ANOVA) was used for NRS at rest and coughing and patients' satisfaction. Categorical data were expressed by $\mathrm{N}(\%)$ and were analyzed with the $\chi^{2}$ test. Fisher's exact test was used for side effects. The value of $p<0.05$ was taken as significant difference. To reduce type I error, $p<0.0083$ was considered statistically significant after Bonferroni correction when comparing two groups.

\section{Results}

During the study, there were no lapses in the blinding. A total of 140 patients were enrolled in this study. Four patients refused to participate before surgery. Four patients were excluded from the study due to alteration of anesthesia drugs or procedures when they occurred hemorrhages or severe hypertensions during operations. Three patients were excluded because of unexpected termination of PCIA after surgery. Five patients were excluded because of incomplete case report form (CRF). Finally, 124 patients undergoing elective gynecological tumors surgery were randomized into four groups: Group $\mathrm{S}(\mathrm{n}=32)$, Group OS ( $=30)$, Group $\mathrm{SO}(\mathrm{n}=30)$ and Group $\mathrm{O}(\mathrm{n}=32)$ (Figure 1). 
There was no statistically significant difference in demographic data including age, gender, BMI, ASA, type of surgery, length of anesthesia, or length of incision in four groups $(p>0.05$, Table 1).

Patients in Group OS and Group O showed shorter time of consciousness recovery and extubation after surgery when compared with patients in Group S and Group SO $(p<$ 0.0083 , Table 2). There was no significant difference in sedation level, rescue analgesia and side effects in PACU in four groups ( $p>0.05$, Table 2). Length of first demand bolus in ward after surgery in Group OS was much longer than that in Group S, and in Group $\mathrm{O}$ was much longer than that in Group SO $(p<0.0083$, Table 2). Accumulated opioid consumption in PCIA (equal to morphine) in Group SO and
Group $\mathrm{O}$ was significantly less than that in Group $\mathrm{S}$ and Group OS $(p<0.0083$, Table 2).

Only one patient in Group $\mathrm{S}$ suffered respiratory depression within 3 hours after surgery (Table 3). PONV were observed in patients from all groups. However, more patients in Group S and Group SO experienced PONV when compared with patients in Group OS and Group O 3 hours after surgery. Differently, more patients in Group S and Group OS experienced PONV when compared with patients in Group SO and Group O while 24 and 48 hours after surgery, but there was no statistical significance ( $p>$ 0.05 , Table 3). There was no significant difference in pruritus or dizziness in four groups at 3, 24 or 48 hours after surgery $(p>0.05$, Table 3$)$.

Table I Comparison of Demographic Data in Patients

\begin{tabular}{|c|c|c|c|c|c|c|c|}
\hline & & Group S $(n=32)$ & Group OS $(n=30)$ & Group SO $(n=30)$ & Group $O(n=32)$ & $F / x^{2}$ & $P$ \\
\hline Age (years) & & $47.84 \pm 9.06$ & $49.47 \pm 10.34$ & $49.00 \pm 8.65$ & $44.03 \pm 9.26$ & 2.18 & 0.094 \\
\hline BMI $\left(\mathrm{kg} / \mathrm{m}^{2}\right)$ & & $24.7 I \pm 3.02$ & $24.38 \pm 3.39$ & $23.01 \pm 3.22$ & $23.75 \pm 2.97$ & 1.73 & 0.165 \\
\hline ASA & I & $9(28.1 \%)$ & $9(30.0 \%)$ & $10(33.3 \%)$ & $15(46.9 \%)$ & 3.02 & 0.388 \\
\hline & II & 23 (7I.9\%) & $21(70.0 \%)$ & $20(66.7 \%)$ & 17 (53.1\%) & & \\
\hline Surgery Types & Laparotomy & $21(65.63 \%)$ & 14 (46.67\%) & $14(46.67 \%)$ & $19(59.4 \%)$ & 3.39 & 0.336 \\
\hline & Endoscopy & II (34.38\%) & $16(53.33 \%)$ & $16(53.33 \%)$ & $13(40.6 \%)$ & & \\
\hline Length of anes & sia (mins) & $158.69 \pm 65.39$ & $159.10 \pm 50.66$ & $|73.17 \pm 4| .49$ & $139.12 \pm 60.45$ & 1.98 & 0.121 \\
\hline Length of incis & $(\mathrm{cm})$ & $|0.09 \pm 5.7|$ & $9.27 \pm 6.53$ & $9.83 \pm 7.51$ & $9.56 \pm 6.08$ & 1.00 & 0.801 \\
\hline
\end{tabular}

Note: Values are expressed as mean \pm SD and the number of patients (\%).

Abbreviations: BMI, body mass index; ASA, American Society of Anesthesiologists; ASA I, a normal healthy patient; ASA II, a patient with mild systemic disease.

Table 2 Comparison of Indicators in Recovery Period, PACU and PCIA

\begin{tabular}{|c|c|c|c|c|c|c|c|}
\hline & & $\begin{array}{l}\text { Group S } \\
(n=32)\end{array}$ & $\begin{array}{l}\text { Group OS } \\
(n=30)\end{array}$ & $\begin{array}{l}\text { Group SO } \\
(n=30)\end{array}$ & $\begin{array}{l}\text { Group } 0 \\
(n=32)\end{array}$ & $\begin{array}{l}\text { F/x } / \\
\text { Wald }\end{array}$ & $P$ \\
\hline \multicolumn{2}{|c|}{$\begin{array}{l}\text { Time of consciousness recovery (Mins) } \\
\text { Time of extubation (Mins) }\end{array}$} & $\begin{array}{l}5.47 \pm 4.27^{\mathrm{a}, \mathrm{c}} \\
7.55 \pm 4.71^{\mathrm{a}, \mathrm{c}}\end{array}$ & $\begin{array}{l}3.65 \pm 2.51^{d} \\
5.24 \pm 3.02^{d}\end{array}$ & $\begin{array}{l}5.64 \pm 2.30^{f} \\
8.34 \pm 2.52^{f}\end{array}$ & $\begin{array}{l}3.82 \pm 2.67 \\
5.35 \pm 2.98\end{array}$ & $\begin{array}{l}6.05 \\
10.43\end{array}$ & $\begin{array}{l}0.00 I^{*} \\
<0.00 I^{* *}\end{array}$ \\
\hline $\begin{array}{l}\text { The Ramsay sedation scale in } \\
\text { PACU (\%) }\end{array}$ & $\begin{array}{l}2 \\
\text { I or } 3\end{array}$ & $\begin{array}{l}26(81.2 \%) \\
6(18.8 \%)\end{array}$ & $\begin{array}{l}27(90.0 \%) \\
3(10.0 \%)\end{array}$ & $\begin{array}{l}24(80.0 \%) \\
6(20.0 \%)\end{array}$ & $\begin{array}{l}29(90.6 \%) \\
3(9.4 \%)\end{array}$ & 2.36 & 0.500 \\
\hline $\begin{array}{l}\text { Rescue analgesia in PACU (\%) } \\
\text { Nausea and vomiting in PACU } \\
\text { Pruritus in PACU } \\
\text { Respiratory depression in PAC }\end{array}$ & & $\begin{array}{l}3(9.4 \%) \\
4(12.5 \%) \\
0 \\
1\end{array}$ & $\begin{array}{l}\text { I }(3.3 \%) \\
\text { I }(3.3 \%) \\
0 \\
0\end{array}$ & $\begin{array}{l}1(3.3 \%) \\
2(6.7 \%) \\
0 \\
0\end{array}$ & $\begin{array}{l}0(0.0 \%) \\
2(6.2 \%) \\
0 \\
0\end{array}$ & $\begin{array}{l}4.45 \\
2.01 \\
- \\
-\end{array}$ & $\begin{array}{l}0.216 \\
0.571 \\
- \\
-\end{array}$ \\
\hline \multicolumn{2}{|c|}{$\begin{array}{l}\text { Length of first demand bolus in ward after } \\
\text { surgery (min) }\end{array}$} & $70(23,240)^{\mathrm{a}}$ & $360(240,600)$ & $140(80,240)^{f}$ & $260(195,600)$ & 18.11 & $<0.001 * *$ \\
\hline \multicolumn{2}{|c|}{$\begin{array}{l}\text { Accumulated opioid consumption in PCIA } \\
\text { (equal to morphine, } \mathrm{mg} \text { ) }\end{array}$} & $120(110,136)^{b, c}$ & $126(|| 6, \mid 40)^{\mathrm{d}, \mathrm{e}}$ & $7(3.5,12)$ & $7(0,16)$ & 92.68 & $<0.00$ I** \\
\hline
\end{tabular}

Notes: Values are expressed as mean \pm SD, the number of patients (\%) and the median $(25 \%, 75 \%)$. ${ }^{a} P<0.0083$, Group $S$ vs Group OS, ${ }^{b} P<0.0083$, Group $S$ vs Group SO, ${ }^{c} P<0.0083$, Group $S$ vs Group O, ${ }^{\mathrm{d} P}<0.0083$, Group OS vs Group SO, ${ }^{\mathrm{e}} P<0.0083$, Group OS vs Group O, ${ }^{f} P<0.0083$, Group SO vs Group O, $* P<0.05$, ${ }^{*} * P<0.001$, vs the four groups. The Ramsay sedation scale, I, anxious or restless or both; 2, cooperative, oriented, and tranquil; 3 , responds to command. 
Table 3 Comparison of Incidence of Side Effects 3, 24 and 48 Hours After Surgery

\begin{tabular}{|l|l|l|l|l|l|}
\hline & Group S (n=32) & Group OS (n=30) & Group SO (n=30) & Group O (n=32) & P \\
\hline Nausea (3 hours) & $7(21.9 \%)$ & $3(10.0 \%)$ & $3(10.0 \%)$ & $4(12.5 \%)$ & 0.499 \\
Nausea (24 hours) & $15(46.9 \%)$ & $12(40.0 \%)$ & $7(23.3 \%)$ & $8(25.0 \%)$ & 0.137 \\
Nausea (48 hours) & $7(21.9 \%)$ & $7(24.1 \%)$ & $3(10.0 \%)$ & $2(6.2 \%)$ & 0.125 \\
Vomiting (3 hours) & $5(15.6 \%)$ & $2(6.7 \%)$ & $2(6.7 \%)$ & $1(3.1 \%)$ & 0.321 \\
Vomiting (24 hours) & $9(28.1 \%)$ & $8(26.7 \%)$ & $5(16.7 \%)$ & $5(15.6 \%)$ & 0.503 \\
Vomiting (48 hours) & $3(9.4 \%)$ & $2(6.9 \%)$ & $3(10.0 \%)$ & $1(3.1 \%)$ & 0.677 \\
Respiratory depression (3 hours) & $1(3.1 \%)$ & 0 & 0 & 0 & 0.186 \\
Respiratory depression (24 hours) & 0 & 0 & 0 & 0 & - \\
Respiratory depression (48 hours) & 0 & 0 & 0 & 0 & - \\
Pruritus (3 hours) & 0 & 0 & 0 & 0 & - \\
Pruritus (24 hours) & 0 & $1(3.3 \%)$ & 0 & 0 & 0.186 \\
Pruritus (48 hours) & 0 & 0 & $1(3.3 \%)$ & 0 & - \\
Dizziness (3 hours) & $I(3.1 \%)$ & 0 & $1(3.3 \%)$ & $2(6.2 \%)$ & 0.866 \\
Dizziness (24 hours) & $3(9.4 \%)$ & $1(3.3 \%)$ & 0 & & 0.835 \\
Dizziness (48 hours) & $3(9.4 \%)$ & $1(3.3 \%)$ & $(3.1 \%)$ & 0.397 \\
\hline
\end{tabular}

Notes: Values are expressed as the number of patients (\%). When $\mathrm{SpO}_{2}$ was $<90 \%$ and respiration rate was $<10$ breath/min, it was documented as respiratory depression.

Although both sufentanil and oxycodone PCIA provided adequate pain relief in ward (NRS $<4$ ), patients in Group OS and Group O showed lower NRS at rest and coughing, but higher patients' satisfaction than patients in Group $\mathrm{S}$ and Group SO 3 hours after surgery $(p<0.0083$, Figure 2A-C). 24 and 48 hours after surgery, patients in Group SO and Group O showed lower NRS at rest and coughing and FAS, but higher patients' satisfaction than patients in Group $\mathrm{S}$ and Group OS ( $p<0.0083$, Figure 2A-C and Table 4$)$. There was no significant difference in the sedation level in four groups $(p>0.05$, Table 4).

Patients in Group SO and Group O showed a shorter time of intestinal recovery, first feeding and first-time movement $(p<0.0083$, Table 4$)$.

\section{Discussion}

PCIA is an efficient way to control postoperative pain, as long as appropriate analgesic is selected and its dose and lockout intervals are properly controlled. ${ }^{11,17}$ Opioids, the most commonly used type of analgesics for PCIA, have a clinical limitation because of side effects such as respiratory depression. ${ }^{18}$ Many studies have investigated the effects of oxycodone for postoperative analgesia, ${ }^{2,16,19-23}$ however it is still controversial whether oxycodone can provide better transition analgesia and PCIA after gynecological tumors surgery under general anesthesia when compared with sufentanil. In our preliminary study, sufentanil PCIA without background infusion was applied; however, the intervals between bonus analgesia were too short (approximately 1 hour), which severely interfered patients' recovery and rest especially in the evening. Differently, oxycodone has much longer half-life (approximately 4 hours) and can provide reasonable intervals between demanded bonus doses during postoperative analgesia. ${ }^{16}$ Therefore, sufentanil PCIA with background infusion and oxycodone PCIA without background infusion were used in this study.

Patients in four groups did not show any difference in rescues analgesia rate in PACU, indicating both oxycodone and sufentanil can provide satisfied transition analgesia, which was consistent with previous studies. ${ }^{24,25}$ However, patients administrated with oxycodone showed relatively shorter time of consciousness recovery and extubation, less incidence rate of PONV, and none occurring of respiratory depression in PACU, demonstrating that, under the similar transitional analgesic effects, oxycodone induced less adverse complications when compared with sufentanil after administration of equal effect does. One possible explanation is the different metabolic productions between sufentanil and oxycodone. Sufentanil is metabolized by the liver and enterocytes of the small intestines, catalyzed by the cytochrome P450 enzyme. Both of its metabolites norsufentanil and demethylsufentanil retain some activities ${ }^{26}$ although the activity of norsufentanil remains low. Differently, the main metabolic productions of oxycodone are noroxycodone and noroxymorphone, the former is a very weak $\mu$-opioid receptor agonist whereas the latter does not contribute significantly to central opioid effects as their plasma concentrations are too low to uptake into the brain 


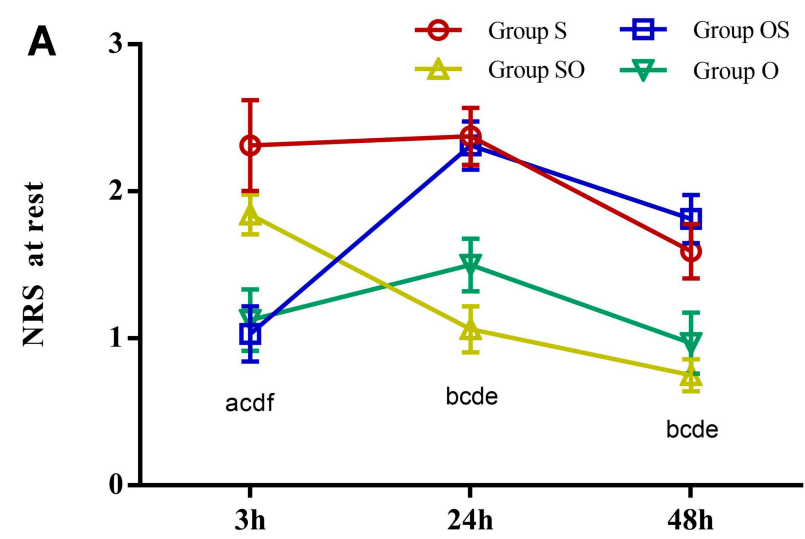

Time after surgery(h)

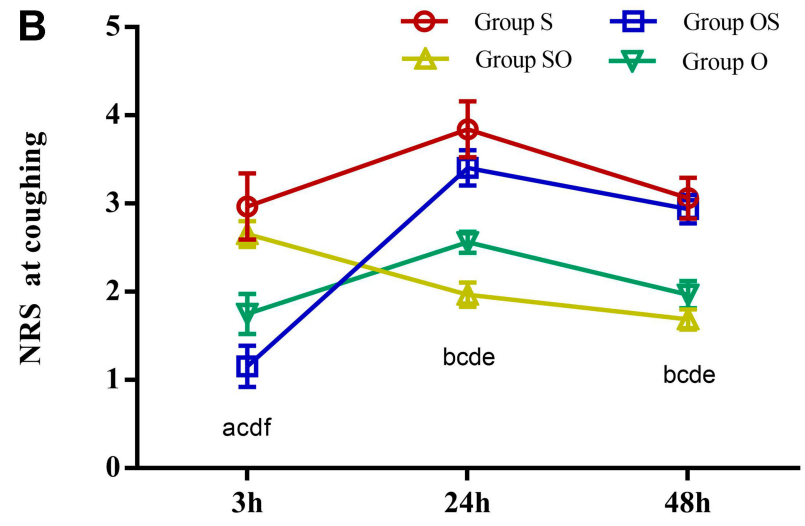

Time after surgery(h)

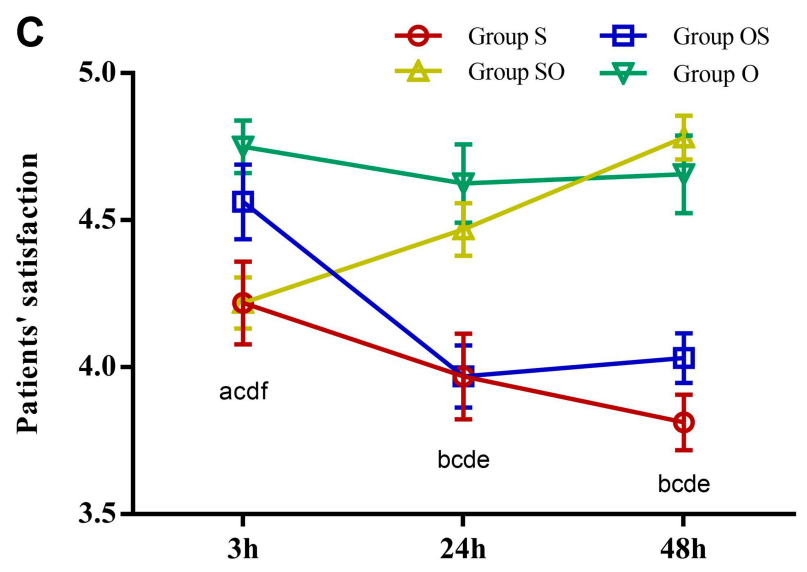

Time after surgery(h)

Figure 2 (A-C) NRS at rest and coughing, and patients' satisfaction 3, 24 and 48 hours after surgery.

Notes: Values are expressed as mean \pm SD. ${ }^{a} P<0.0083$, Group $S$ vs Group OS, ${ }^{b} P<0.0083$, Group $S$ vs Group SO, ${ }^{c} P<0.0083$, Group $S$ vs Group $O$, ${ }^{\text {d }} P<0.0083$, Group OS vs Group SO, ${ }^{e} P<0.0083$, Group OS vs Group $O,{ }^{f} P<0.0083$, Group SO vs Group $O$ comparing groups.

Abbreviations: NRS, Numerical Rating Scale, with 0-10, 0 indicating no pain and 10 indicating unbearable pain. Patients' satisfaction was measured on a 5-point scale ( 1 , very dissatisfied; 2 , dissatisfied; 3 , neutral; 4 , satisfied; 5 , very satisfied)

although it is a more potent agonist than oxycodone. ${ }^{27}$ Noticeably, on the basis of the same PCIA, length of first demand bolus in ward after surgery from patients received oxycodone in transition analgesia was much longer than patients received sufentanil in transition analgesia, demonstrating that the longer half-life of oxycodone could provide prolong intervals for demanded bolus which may increase the patient's satisfaction.

After returning to wards, both sufentanil and oxycodone PCIA provided adequate postoperative pain analgesia (NRS $<4)$. However, accumulated opioid consumption in patients with oxycodone PCIA (equal to morphine) was significantly less than that in patients with sufentanil PCIA. There are two possible explanations to this result. One is that oxycodone has more effective in visceral pain than other opioids. Oxycodone binds to both $\mu$-and $\kappa$-opioid receptors, and thereby alleviated not only somatic but also visceral pain, consequently has high therapeutic efficacy in acute postoperative pain after abdominal surgery. ${ }^{28,29}$ This is further confirmed by the lower FAS scores and higher patients' satisfaction in oxycodone PCIA patients when compared with those with sufentanil PCIA. The other reason is different infusion patterns between oxycodone and sufentanil PCIA. Different with sufentanil which need background infusion, no background infusion was administered in oxycodone PCIA due to its much longer half-life clearance, which significantly reduces the doses of analgesic during postoperative pain relief treatment. The reduced analgesic does bring further benefit for postoperative recovery. For example, patients with oxycodone PCIA showed a shorter time of intestinal recovery, first feeding and first-time movement. The lack of a significant difference in sedation level in four groups probably due to the 
Table 4 Comparison of Indicators 3, 24 and 48 Hours After Surgery in Ward

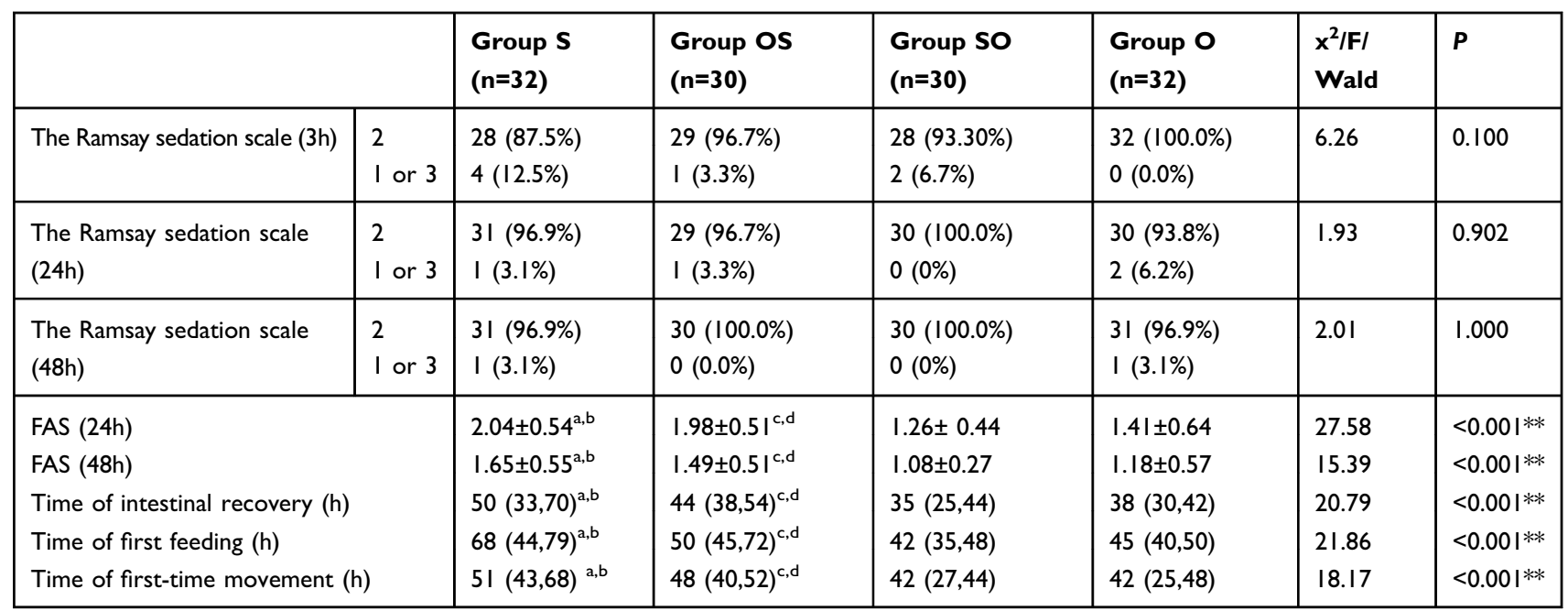

Notes: Values are expressed as the number of patients (\%), mean \pm SD and the median ( $25 \%, 75 \%)$. ${ }^{a} p<0.0083$, Group $S$ vs Group SO, ${ }^{b} p<0.0083$, Group $S$ vs Group $O$, ${ }^{c} p<0.0083$, Group OS vs Group SO, ${ }^{d} p<0.0083$, Group OS vs Group $O,{ }^{*} * P<0.001$, vs the four groups. The Ramsay sedation scale, 1 , anxious or restless or both; 2 , cooperative, oriented, and tranquil; 3, responds to command; FAS, I, unrestricted activity; 2, partially restricted activity; 3, severely restricted activity.

Abbreviations: FAS, Functional activity score

limited number of sample size and enrolled patients who were $\leq 65$ years of age and had an ASA physical status of I or II in this study.

The most common postoperative side effect of PCIA is PONV. The high-risk factors for PONV include gender (female), non-smoking patients, a history of PONV, and the use of opioid during or after surgery. The occurrence of PONV in the patients presenting a high-risk factor is $21 \%, 39 \%$ in those with two risk factors, $61 \%$ in those with three, and $79 \%$ in those with four risk factors. ${ }^{30}$ Administration of a preventive antiemetic drug is recommended for patients at high risk. In our study, considering all patients had at least 2 risk factors, $5 \mathrm{mg}$ dexamethasone and $0.3 \mathrm{mg}$ of tropisetron were injected intravenously as a PONV preventive antiemetic. Nevertheless, PONV were still observed in patients in all groups, and the incidence rate of PONV was more than 20\% 24 hours after surgery, which is similar to previous studies. ${ }^{31,32}$ However, patients with sufentanil PCIA showed higher incidence rate of PONV when compared with patients with oxycodone PCIA 24 and 48 hours after surgery, demonstrating the advantages of oxycodone over sufentanil in postoperative pain relief. Respiratory depression is one of the most concerned anesthesia-related adverse complications after surgery. Only one patient in Group $\mathrm{S}$ suffered respiratory depression within 3 hours after surgery. We deduced that this was mainly caused by higher does consumption in PCIA in relative to other groups due to their different delivery patterns. However, another possible explanation was that, patients from sufentanil groups may require more bolus does because they due their discomforted feeling of visceral pain, leading to the overdoes of sufentanil, consequently inducing respiratory depression. Moreover, the possible influence of genetic variations cannot be ignored ${ }^{33}$ although we did not perform the genotype analysis. It is estimated that 8 $10 \%$ possibility of a cytochrome P450 enzyme deficiency causes an inability to metabolize the prodrug into the active component and thereby increasing the adverse effects. However, it is too early to say oxycodone is safer than sufentanil due to the limited number of sample size in this study.

There are some limitations in this study. Firstly, our results only represent the analgesic effects and adverse reactions of oxycodone and sufentanil under fixed dosage and PCIA after gynecological tumor surgery. Further research on different surgery, more doses and kinds of pathway in PCIA is needed to estimate if oxycodone is superior to sufentanil in postoperative analgesia. Secondly, the study was performed in only one hospital with limited patients enrolled, larger scale clinical trial with multiple centers is needed in the future.

\section{Conclusion}

Both oxycodone and sufentanil provided adequate pain relief in transitional analgesia and PCIA treatment after surgery. Oxycodone without background infusion showed less analgesic drug consumption and faster recovery than 
sufentanil with background infusion in PCIA after gynecological tumor operation under general anesthesia.

\section{Data Sharing Statement}

The authors will allow sharing of participant data. The data will be available for anyone who wishes to access them for any purpose. The data will be accessible from immediately following publication to 6 months after publication, and contact should be made via the first author by email.

\section{Ethical Statement}

The authors declare that all the patients provided written informed consent and that this study was conducted in accordance with the Declaration of Helsinki. This study was approved by the clinical research ethics committee of Shaanxi Provincial Cancer Hospital (No. 2017-07), and was registered in Chinese Clinical Trial Registry (registration number ChiCTR-IOR-1800014793).

\section{Acknowledgments}

This work was supported by basic research program of Natural Science in Shaanxi Province (grant no. 2018JM7038) and program in the field of social development at the Department of Science and Technology in Shaanxi Province (grant nos. 2018SF-038, 2018SF-010).

\section{Author Contributions}

Sha-Jie Dang and Rui-Li Li helped with writing the original draft and data analysis, Jun Wang, Wen-Bin Zeng, Yun He and Hui-Yu Yue helped with patient recruitment and data collection, Li-chun Han helped with the study design, supervision and validation, and Si-Yuan Li also helped with directing the study project and revised and edited the manuscript. All authors contributed to data analysis, drafting or revising the article, gave final approval of the version to be published, and agree to be accountable for all aspects of the work.

\section{Disclosure}

The authors report no conflicts of interest in this work.

\section{References}

1. Wu CL, Raja SN. Treatment of acute postoperative pain. Lancet. 2011;377(9784):2215-2225. doi:10.1016/S0140-6736(11)60245-6

2. Boo-Young H, Jae-Young K, Eunsoo K, Do-Won L, Tae-Kyun K, Hae-Kyu $\mathrm{K}$. Oxycodone vs. fentanyl patient-controlled analgesia after laparoscopic cholecystectomy. Int J Med Sci. 2014;11(7):658-662. doi:10.7150/ijms.8331

3. Morlion B, Schäfer M, Betteridge N, Kalso E. Non-invasive patientcontrolled analgesia in the management of acute postoperative pain in the hospital setting. Curr Med Res Opin. 2018;34(7):1-17. doi:10.1080/03007995.2018.1462785
4. Chou R, Gordon DB, Leon-Casasola OAD, et al. Management of postoperative pain: a clinical practice guideline from the American pain society, the American society of regional anesthesia and pain medicine, and the American society of anesthesiologists' committee on regional anesthesia, executive commit. J Pain. 2016;17(2):131157. doi:10.1016/j.jpain.2015.12.008

5. George JA, Lin EM, Hanna, MD MN. The effect of intravenous opioid patient-controlled analgesia with and without background infusion on respiratory depression: a meta-analysis. $J$ Opioid Manag. 2010;6(1):47-54. doi:10.5055/jom.2010.0004

6. Hong PT, Conroy T. The effectiveness of intravenous oxycodone in the treatment of acute postoperative pain: a systematic review. $J$ Perianesth Nurs. 2017;S1089947217303246.

7. Joong-Ho P, Chiu L, Youngmin S, Ji-Hyun A, Jong-Seouk B, Ji-Hyang L. Comparison of oxycodone and fentanyl for postoperative patientcontrolled analgesia after laparoscopic gynecological surgery. Korean $J$ Anesthesiol. 2015;68(2):153-158. doi:10.4097/kjae.2015.68.2.153

8. Kim NS, Lee JS, Park SY, et al. Oxycodone versus fentanyl for intravenous patient-controlled analgesia after laparoscopic supracervical hysterectomy: a prospective, randomized, double-blind study. Medicine. 2017;96(10):e6286. doi:10.1097/MD.0000000000006286

9. Nie J, Sun S, Huang S. Effect of oxycodone patient-controlled intravenous analgesia after cesarean section: a randomized controlled study. J Pain Res. 2017;10:2649-2655. doi:10.2147/JPR.S142896

10. Raff M, Belbachir A, El-Tallawy S, et al. Intravenous oxycodone versus other intravenous strong opioids for acute postoperative pain control: a systematic review of randomized controlled Trials. Pain Ther. 2019;8(1):19-39. doi:10.1007/s40122-019-0122-4

11. Zhang B, Wang G, Liu X, Wang TL, Chi P. The opioid-sparing effect of perioperative dexmedetomidine combined with oxycodone infusion during open hepatectomy: a randomized controlled trial. Front Pharmacol. 2017;8:940. doi:10.3389/fphar.2017.00940

12. Piirainen P, Kokki H, Hautajärvi H, Ranta VP, Kokki M. The analgesic efficacy and pharmacokinetics of epidural oxycodone after gynaecologic laparotomy - a randomised, double-blind, double-dummy comparison with intravenous administration. Br J Clin Pharmacol. 2018;84(9):2088-2096. doi:10.1111/bcp.13643

13. Xiang X, Yuan X, Lian Y, Fang J, Wu Y. Effect of oxycodone hydrochloride combined with flurbiprofen axetil for intravenous patient-controlled analgesia in lower abdominal patients: a randomized trial. Medicine. 2018;97(7):e9911. doi:10.1097/MD.0000000000009911

14. Juncheng X, Xiang C, Chengwei W, Shuqun L, Jian L. Intra-operative oxycodone reduced postoperative catheter-related bladder discomfort undergoing transurethral resection prostate. a prospective, double blind randomized study. Urol J. 2019.

15. Chang SH, Maney KM, Phillips JP, Langford RM, Mehta VA. comparison of the respiratory effects of oxycodone versus morphine: a randomised, double-blind, placebo-controlled investigation. Anaesthesia. 2010;65(10):1007-1012. doi:10.1111/j.1365-2044.2010.06498.x

16. Wang N, Zhou H, Song X, Wang J. Comparison of oxycodone and sufentanil for patient-controlled intravenous analgesia after laparoscopic radical gastrectomy: a randomized double-blind clinical trial. Anesth Essays Res. 2016;10(3):557-560. doi:10.4103/0259-1162.186603

17. Kim NS, Kang KS, Yoo SH, et al. A comparison of oxycodone and fentanyl in intravenous patient-controlled analgesia after laparoscopic hysterectomy. Korean J Anesthesiol. 2015;68(3):261-266. doi:10.4097/kjae.2015.68.3.261

18. Grass JA. Patient-controlled analgesia. Anesth Analg. 2005;101 (Supplement):S44-61. doi:10.1213/01.ANE.0000177102.11682.20

19. Ding Z, Wang K, Wang B, Zhou N, Li H, Yan B. Efficacy and tolerability of oxycodone versus fentanyl for intravenous patientcontrolled analgesia after gastrointestinal laparotomy. Medicine. 2016;95(39):e4943. doi:10.1097/MD.0000000000004943

20. Xie K, Zhang W, Fang W, Lian Y, Lin S, Fang J. The analgesic efficacy of oxycodone hydrochloride versus fentanyl during outpatient artificial abortion operation: a randomized trial. Medicine. 2017;96(26):e7376. doi:10.1097/MD.0000000000007376 
21. Quan Z, Luo C, Chi P, Wang L, He H. Analgesic effects of oxycodone relative to those of sufentanil, in the presence of midazolam, during endoscopic injection sclerotherapy for patients with cirrhosis and esophageal varices. Anesth Analg. 2018;1.

22. An Y, Zhao L, Wang T, et al. Preemptive oxycodone is superior to equal dose of sufentanil to reduce visceral pain and inflammatory markers after surgery: a randomized controlled trail. BMC Anesthesiol Electron Resour. 2019;19(1):96. doi:10.1186/s12871-019-0775-x

23. Kwon YS, Jang JS, Lee NR, et al. A comparison of oxycodone and alfentanil in intravenous patient-controlled analgesia with a timescheduled decremental infusion after laparoscopic cholecystectomy. Pain Res Manag. 2016;2016:7868152. doi:10.1155/2016/7868152

24. Cajanus K, Kaunisto MA, Tallgren M, Jokela R, Kalso E. How much oxycodone is needed for adequate analgesia after breast cancer surgery: effect of the OPRM1 118A >G polymorphism. J Pain. 2014;15 (12):1248-1256. doi:10.1016/j.jpain.2014.09.002

25. Han L, Su Y, Xiong H, et al. Oxycodone versus sufentanil in adult patient-controlled intravenous analgesia after abdominal surgery: a prospective, randomized, double-blinded, multiple-center clinical trial. Medicine. 2018;97(31):e11552. doi:10.1097/MD.0000000000011552

26. Wilde M, Pichini S, Pacifici R. Metabolic pathways and potencies of new fentanyl analogs. Front Pharmacol. 2019;10:238. doi:10.3389/ fphar.2019.00238
27. Kokki M, Valitalo P, Kuusisto M, et al. Central nervous system penetration of oxycodone after intravenous and epidural administration. Br J Anaesth. 2014;112(1):133-140. doi:10.1093/bja/aet337

28. Kokki H, Kokki M, Sjovall S. Oxycodone for the treatment of postoperative pain. Expert Opin Pharmacother. 2012;13(7):1045-1058. doi: $10.1517 / 14656566.2012 .677823$

29. Gebhart GF, Bielefeldt K. Physiology of Visceral Pain. Compr Physiol. 2016;6:1609-1633.

30. Apfel CC, Läärä E, Koivuranta M, Greim CA, Roewer N. A simplified risk score for predicting postoperative nausea and vomiting: conclusions from cross-validations between two centers. Anesthesiology. 1999;91 (3):693-700. doi:10.1097/00000542-199909000-00022

31. Dong CS, Zhang J, Lu Q, et al. Effect of dexmedetomidine combined with sufentanil for post- thoracotomy intravenous analgesia: a randomized, controlled clinical study. BMC Anesthesiol. 2017;17(1):33. doi:10.1186/s12871-017-0324-4

32. Tao B, Liu K, Wang D, Ding M, Zhao P. Effect of intravenous oxycodone versus sufentanil on the incidence of postoperative nausea and vomiting in patients undergoing gynecological laparoscopic surgery. $J$ Clin Pharmacol. 2019;59(8):1144-1150. doi:10.1002/jcph.1408

33. Orliaguet G, Hamza J, Couloigner V, et al. A case of respiratory depression in a child with ultrarapid CYP2D6 metabolism after tramadol. Pediatrics. 2015;135(3):e753-755. doi:10.1542/peds.2014-2673
Journal of Pain Research

\section{Publish your work in this journal}

The Journal of Pain Research is an international, peer reviewed, open access, online journal that welcomes laboratory and clinical findings in the fields of pain research and the prevention and management of pain. Original research, reviews, symposium reports, hypothesis formation and commentaries are all considered for publication. The manuscript management system is completely online and includes a very quick and fair peer-review system, which is all easy to use. Visit http:// www.dovepress.com/testimonials.php to read real quotes from published authors. 